\title{
Strangeness Production in Proton-Proton Collisions Close to Threshold*
}

\author{
M. Wolke ${ }^{\dagger}$, H.-H. Adam ${ }^{\mathrm{b}}$, A. Budzanowski ${ }^{\mathrm{c}}$, R. Czyżykiewicz ${ }^{\mathrm{d}}$, D. Grzonka ${ }^{\mathrm{a}}$, \\ M. Janusz ${ }^{\mathrm{d}}$, L. Jarczyk ${ }^{\mathrm{d}}$, B. Kamys ${ }^{\mathrm{d}}$, A. Khoukaz ${ }^{\mathrm{b}}$, K. Kilian ${ }^{\mathrm{a}}$, P. Kowina ${ }^{\mathrm{a}}$, T. Lister ${ }^{\mathrm{b}}$, \\ P. Moskal ${ }^{\text {ad }}$, W. Oelert ${ }^{\mathrm{a}}$, T. Rożek ${ }^{\mathrm{ae}}$, R. Santo ${ }^{\mathrm{b}}$, G. Schepers ${ }^{\mathrm{a}}$, T. Sefzick ${ }^{\mathrm{a}}$, \\ M. Siemaszko e , J. Smyrski ${ }^{d}$, S. Steltenkamp ${ }^{b}$, A. Strzałkowski ${ }^{d}$, P. Winter ${ }^{a}$, \\ P. Wüstner ${ }^{\mathrm{a}}$, W. Zipper \\ ${ }^{a}$ IKP \& ZEL, Forschungszentrum Jülich, D-52425 Jülich, Germany \\ bIKP, Westfälische Wilhelms-Universität, D-48149 Münster, Germany \\ ' Institute of Nuclear Physics, PL-31-342 Cracow, Poland \\ dinstitute of Physics, Jagellonian University, PL-30-059 Cracow, Poland \\ e Institute of Physics, University of Silesia, PL-40-007 Katowice, Poland
}

Exclusive data on the reactions $p p \rightarrow p p K^{+} K^{-}$and $p p \rightarrow p K^{+} \Lambda / \Sigma^{0}$ have been taken at the cooler synchrotron COSY close to threshold.

At equal excess energies, an enhancement of the $\Lambda / \Sigma^{0}$ ratio by one order of magnitude has been observed compared to data at higher excess energies. New results obtained at the COSY-11 facility explore the transition region between this low-energy $\Sigma^{0}$ suppression and excess energies of $60 \mathrm{MeV}$.

A first total cross section for elementary antikaon production below the $\phi$ threshold has been determined, two orders of magnitude smaller compared to kaon production at the same excess energy.

\section{ELEMENTARY ANTIKAON PRODUCTION}

Studies on the reaction $p p \rightarrow p p K^{+} K^{-}$close to threshold have been motivated by the continuing discussion on the nature of the scalar resonances $f_{0}(980)$ and $a_{0}(980)$ [ 1]. Within the Jülich meson exchange model the $K \bar{K}$ interaction gives rise to a bound state in the isoscalar sector identified with the $f_{0}(980)$ [ 2]. Both shape and absolute scale of $\pi \pi \rightarrow K \bar{K}$ transitions crucially depend on the strength of the $K \bar{K}$ interaction, which in turn is a prerequisite of a $K \bar{K}$ molecule interpretation of the $f_{0}(980)$. Similar effects might be expected in proton-proton scattering, and first results of exploratory microscopic calculations have recently been presented [3].

\footnotetext{
${ }^{*}$ Partly supported by the European Community - Access to Research Infrastructure action of the Improving Human Potential Programme.

${ }^{\dagger}$ present address: The Svedberg Laboratory, Thunbergsvägen 5A, Box 533, S-75121 Uppsala, Sweden.

${ }^{\ddagger}$ talk given by P. Moskal.
} 
A first total cross section value for the elementary antikaon production below the $\phi$ threshold in proton-proton scattering has been extracted from exclusive data taken at the COSY-11 installation at an excess energy of $\mathrm{Q}=17 \mathrm{MeV}$ with $\sigma=1.80 \pm 0.27_{-0.35}^{+0.28} \mathrm{nb}$ including statistical and systematical errors, respectively [4. The experimental technique is based on the measurement of the complete four-momenta of positively charged ejectiles. Requiring furthermore a $K^{-}$consistent hit in the dedicated negative particle detection system of the COSY-11 facility [ 5], the identification of the four particle final state becomes (almost) completely free of background (fig. 11).

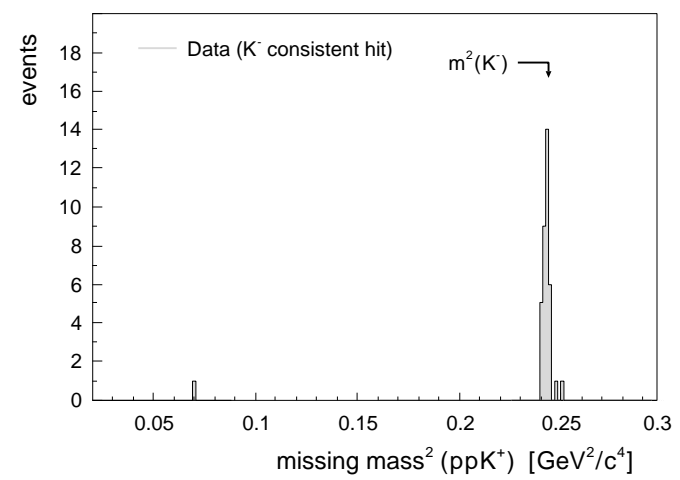

Figure 1. Missing mass squared with respect to an identified $\left(p p K^{+}\right)$subsystem $17 \mathrm{MeV}$ above the $K^{+} K^{-}$threshold. The bin width corresponds to an experimental resolution of $\approx 2 \mathrm{MeV} / \mathrm{c}^{2}(\mathrm{FWHM})$.

However, the presently available statistics of $K^{+} K^{-}$events is not sufficient to distinguish non-resonant $K^{+} K^{-}$production and resonant production via the scalar resonances $f_{0}(980)$ and $a_{0}(980)$ from differential observables, e.g. the $K \bar{K}$ invariant mass distribution.

Considering the energy dependence of the total cross section, $\eta, \omega$ and $\eta^{\prime}$ production indicate strong imprints of final state interaction (FSI) at excess energies $\mathrm{Q} \leq 100 \mathrm{MeV}$ in the proton-proton and, in case of $\eta$, in the proton-meson subsystems. Contrary to this, $p p \rightarrow p p K^{+} K^{-}$cross section data obtained at COSY-11 [4] and DISTO [6] below and above the $\phi$ threshold, respectively, are in reasonable agreement with one-boson exchange calculations [7] neglecting FSI effects. Presently it is not clear whether the absence of the FSI influence in the $p p \rightarrow p p K^{+} K^{-}$reaction might be explained by a partial compensation of the $p p$ and $K^{-} p$ interaction in the final state or by the additional degree of freedom given by the four-body final state. In the latter case FSI effects are expected to be more pronounced at energies very close to the $K^{+} K^{-}$production threshold [ 8].

Data taking at excess energies closer to threshold and slightly below the $\Phi$ production threshold, i.e. at excess energies of $10 \mathrm{MeV}$ and $28 \mathrm{MeV}$ with respect to the $K^{+} K^{-}$ threshold, has been successfully completed early this year at the COSY-11 facility and data analysis is presently in progress.

\section{EXCLUSIVE KAON-HYPERON FINAL STATES}

The most striking feature of the exclusive close-to-threshold data on $\Lambda$ and $\Sigma^{0}$ production in proton-proton scattering taken at the COSY-11 facility [ 9, 10] is the $\Sigma^{0}$ suppression with $\sigma\left(p p \rightarrow p K^{+} \Lambda\right) / \sigma\left(p p \rightarrow p K^{+} \Sigma^{0}\right)=28_{-9}^{+6}$ observed at equal excess energies below $\mathrm{Q}=13 \mathrm{MeV}$. At excess energies $\geq 300 \mathrm{MeV}$ this ratio is known to be about $2.5[11]$.

Inclusive $K^{+}$production data in $p p$ scattering from the SPES 4 facility at an excess energy of $252 \mathrm{MeV}$ with respect to the $p K^{+} \Lambda$ threshold show enhancements at the $\Lambda p$ 
and $\Sigma N$ thresholds of similar magnitude [12]. Qualitatively, a strong $\Sigma N \rightarrow \Lambda p$ final state conversion might account for both the inclusive SATURNE results as well as the $\Sigma^{0}$ depletion in the COSY-11 data. Evidence for such conversion effects is known e.g. from exclusive hyperon data via $K^{-} d \rightarrow \pi^{-} \Lambda p[13]$.

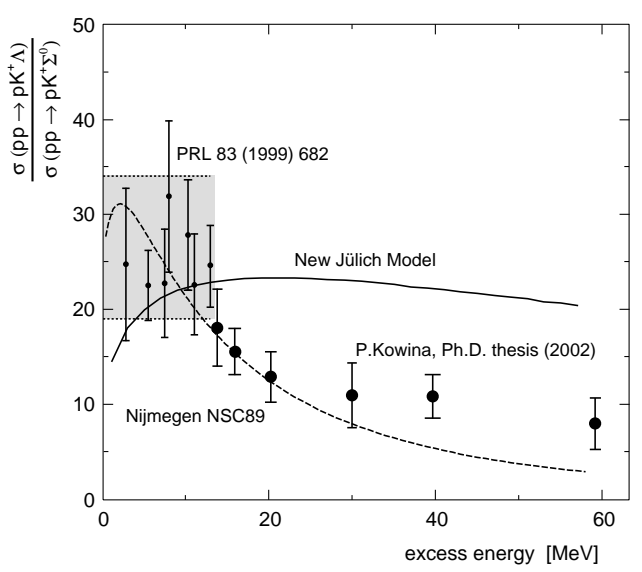

Figure 2. $\Lambda / \Sigma^{0}$ production ratio in protonproton collisions as a function of the excess energy. Data within the shaded area are from [ 10], results at higher excess energies from [ 18]. Calculations [19] within the Jülich meson exchange model assume a destructive interference of $K$ and $\pi$ exchange and employ the microscopic $Y N$ interaction models Nijmegen NSC89 (dashed line [20]) and the new Jülich model (solid line [21]), respectively.

However, in exploratory calculations performed within the framework of the Jülich meson exchange model [14], taking into account both $\pi$ and $K$ exchange diagrams in a coupled channel approach, a final state conversion is rather excluded as origin of the experimentally observed ratio. While $\Lambda$ production is found to be dominated by kaon exchange both $\pi$ and $K$ exchange turn out to contribute to the $\Sigma^{0}$ channel with similar strength. It is concluded [14], that a destructive interference of $\pi$ and $K$ exchange might explain the close-to-threshold $\Sigma^{0}$ suppression.

An experimental study of $\Sigma$ production in different isospin configurations should provide a crucial test for the above interpretation, as for the reaction $p p \rightarrow n K^{+} \Sigma^{+}$an opposite interference pattern is found as compared to the $p K^{+} \Sigma^{0}$ channel. Measurements close to threshold are planned at the COSY-11 facility in the near future.

Contributions from direct production as well as heavy meson exchanges have been neglected so far in these calculations [14] but might influence the $\Lambda / \Sigma^{0}$ production ratio [ 15]. For complementary theoretical studies - considering strangeness production close to threshold to proceed by one-boson exchanges or one-boson exchange followed by the excitation of nucleon resonances — we refer to refs. [16] and a recent review [17].

Measurements on the $\Lambda / \Sigma^{0}$ production ratio in proton-proton collisions have been extended up to excess energies of $\mathrm{Q}=60 \mathrm{MeV}$ at the COSY-11 installation [ 18. In comparison to the experimental data, in figure 2 calculations are included obtained within the approach of [ 14] assuming a destructive interference of $\pi$ and $K$ exchange with different choices of the microscopic hyperon nucleon model to describe the interaction in the final state [19]. The result depends on the details - especially the off-shell properties - of the hyperon-nucleon interaction employed. At the present stage both the good agreement found in [14] for Jülich model A [22] with the close-to-threshold result and for the Nijmegen model (dashed line in fig. 21) with the energy dependence of the cross section ratio should rather be regarded as accidental ${ }^{4}$. Calculations using the new Jülich model (solid line in fig. 2 [21]) do not reproduce the tendency of the experimental

\footnotetext{
${ }^{4}$ In the latter case an $\mathrm{SU}(2)$ breaking in the ${ }^{3} \mathrm{~S}_{1} \Sigma N$ channel had to be introduced [20] resulting in an ambiguity for the $\Sigma^{0} p$ amplitude [23].
} 
data. It is suggested in [19] that neglecting the energy dependence of the elementary amplitudes and higher partial waves might no longer be justified beyond excess energies of $20 \mathrm{MeV}$.

The energy dependence of the total cross section for $\Lambda$ production up to excess energies of $\mathrm{Q}=60 \mathrm{MeV}$ is much better described by a phase space behaviour modified by the $p \Lambda$ final state interaction than by pure phase space [18. However, unlike the findings of [ [10] based on data up to $\mathrm{Q}=13 \mathrm{MeV}$, in the energy range up to $60 \mathrm{MeV} \Sigma^{0}$ production is equally well described neglecting any FSI effect. One reason for this qualitatively different behaviour might be, that the $\Sigma^{0} p$ FSI is much weaker compared to the $\Lambda_{-}^{-}$ proton system. On the other hand, a fit to the energy dependence based on a phase space behaviour implies dominant $\mathrm{S}$-wave production and energy independent reaction dynamics as discussed above. Within the statistics of the present experiment, $\mathrm{P}$-wave contributions can be neither ruled out nor confirmed at higher excess energies for $\Sigma^{0}$ production. Consequently, high statistics $\Sigma^{0}$ data would be needed in future to study the onset of different partial waves experimentally.

\section{REFERENCES}

1. D. Morgan, M.R. Pennington, Phys. Rev. D 48 (1993) 1185; F. Kleefeld et al., Phys. Rev. D 66 (2002) 034007; R.L. Jaffe, Phys. Rev. D 15 (1977) 267; F.E. Close, Rep. Prog. Phys. 51 (1988) 833; F.E. Close et al., Phys. Lett. B 319 (1993) 291; J. Weinstein, N. Isgur, Phys. Rev. D 41 (1990) 2236; D. Lohse et al., Nucl. Phys. A 516 (1990) 513; Z.S. Wang et al., Nucl. Phys. A 684 (2000) 429c.

2. O. Krehl et al., Phys. Lett. B 390 (1997) 23.

3. J. Haidenbauer, Publ. of Research Centre Jülich: Matter and Materials 11 (2002) 225.

4. C. Quentmeier et al., Phys. Lett. B 515 (2001) 276.

5. S. Brauksiepe et al., Nucl. Instr. \& Meth. A 376 (1996) 397.

6. F. Balestra et al., Phys. Rev. C 63 (2001) 024004.

7. A. Sibirtsev et al., Z. Phys. A 358 (1997) 101.

8. A. Sibirtsev, Publ. of Research Centre Jülich: Matter and Materials 11 (2002) 239.

9. J.T. Balewski et al., Phys. Lett. B 420 (1998) 211.

10. S. Sewerin et al., Phys. Rev. Lett. 83 (1999) 682.

11. A. Baldini et al., Total Cross-Sections for Reactions of High-Energy Particles, Springer, Berlin, 1988.

12. R. Siebert et al., Nucl. Phys. A 567 (1994) 819.

13. T.H. Tan, Phys. Rev. Lett. 23 (1969) 395.

14. A. Gasparian et al., Phys. Lett. B 480 (2000) 273.

15. N. Kaiser, Eur. Phys. J. A 5 (1999) 105.

16. K. Tsushima et al., Phys. Rev. C 59 (1999) 369; A. Sibirtsev et al., e-Print Archive nucl-th/0004022 (2000); J.M. Laget, Nucl. Phys. A 691 (2001) 11c; R. Shyam et al., Phys. Rev. C 63 (2001) 022202.

17. P. Moskal et al., Prog. Part. Nucl. Phys. 49 (2002) 1.

18. P. Kowina, Ph.D. thesis, Silesian University Katowice (2002).

19. A. Gasparyan, Publ. of Research Centre Jülich: Matter and Materials 11 (2002) 205.

20. P.M.M. Maessen et al., Phys. Rev. C 40 (1989) 2226.

21. J. Haidenbauer et al., AIP Conf. Proc. 603 (2001) 421. 
22. B. Holzenkamp et al., Nucl. Phys. A 500 (1989) 485.

23. J. Haidenbauer, private communications. 